\title{
Does psychological capital moderate the relationship between worries about accidents and sleepiness?
}

\author{
Kjersti Bergheim Valdersnes ${ }^{1}$, Jarle Eid ${ }^{1}$, Sigurd William Hystad ${ }^{1}$, Morten Birkeland Nielsen ${ }^{1,2}$ \\ ${ }^{1}$ Department of Psychosocial Science, Bergen, Norway \\ ${ }^{2}$ National Institute of Occupational Health, Oslo, Norway
}

\begin{abstract}
The present study investigated psychological capital (PsyCap) as a protective factor in the relationship between worries about accidents and sleepiness among seafarers. The hypothesis that strong PsyCap weakens the relationship between worries about accidents and sleepiness was tested in a cross-sectional sample of 397 maritime workers. In contrast to expectations, the findings indicated a reverse buffering effect in that PsyCap only had a protective impact on sleepiness when worries about accidents were low. For workers that were highly worried, a strong PsyCap was associated with increased levels of sleepiness. The established associations remained consistent after controlling for workers' years of experience as seafarers, and their ratings of psychological safety climate. An interpretation of this finding is that seafarers with high levels of PsyCap will be attentive when the threat level is serious, but will not be bothered when exposed to everyday strain and hassles associated with their work situation.
\end{abstract}

(Int Marit Health 2017; 68, 4: 245-251)

Key words: psychological capital, psychological safety climate, risk perception, sleep quality, maritime

\section{INTRODUCTION}

Seafaring is a dangerous occupation due to the combination of several physical and psychosocial exposures and hazards [1, 2]. With regard to physical risk factors, seafarers are expected to perform safety critical tasks despite changing temperatures, various weather conditions, noise, vibrations and movements of the ship. As for psychosocial risks, seafarers work in 24-7 shift work schedules and are separated from family and friends for prolonged periods. Ideally, workers in high-risk occupations, such as seafaring, should be vigilant and well rested. This is not always the case when workers are worried and concerned about safety issues at work. Actually, stress is considered the primary cause of persistent psychophysiological insomnia [3], and both theoretical models and existing empirical evidence suggest that work-related stress and worries disturb sleep [3-6]. For instance, Lichtstein and Rosenthal [7] found that individuals with insomnia were ten times more likely to attribute their sleep disturbances to cognitive factors, including worrying, than to somatic complaints. Further, it has been argued that physiological and psychological responses to work-related exposures are incommensurate with the deactivation that characterizes sleep $[3,8]$.

However, workers do not necessarily react to exposures and worries to the same degree. Following from Lazarus and Folkman's [9] transactional model of stress and coping, the consequences of environmental stressors are dependent upon how the individual interprets and judges the threat, as well as on the resources the individual has at his or her disposal to deal with the threat. One newly established individual resource for coping with potentially challenging work life situations is psychological capital (PsyCap) [10-12]. PsyCap is a higher order construct that consists of the core elements: self-efficacy, optimism, hope and resiliency. Individuals with a high self-efficacy will generally have stronger beliefs in their ability to control outcomes and succeed in addressing more difficult challenges than those low in efficacy [13]. Optimistic workers will be more likely to build positive outcome expectancies that help them deal with difficult situations [14]. Individuals with a higher level of hope will show greater goal-directed energy and be more likely to find alternative ways to accomplish their goals [15]. Finally, people with a higher score on resiliency tend

Prof. Morten Birkeland Nielsen, Department of Psychosocial Science, Postboks 7807, 5020 Bergen, Norway, tel/fax: 004755582335, e-mail: morten.nielsen@stami.no 
to adapt better when they experience changes or setbacks [16]. These factors can be measured, developed and maintained for performance improvement in the workplace [11]. Findings show that PsyCap has a direct positive association with safety, explaining about $20 \%$ of the variance in safety perceptions among maritime workers [17]. With regard to PsyCap as a protective resource, a study by Schaubroeck and colleagues [18] found that PsyCap plays an important role in differentiating between people who are more or less adaptive to extremely stressful environments. Hence, there are reasons to expect that PsyCap can be a potential moderator of the outcomes of work-related stressors.

Despite the importance of the maritime industry around the globe, there is a lack of studies that have examined how stressful work exposures are related to workers' well-being in seafaring. Adding to the current knowledge base, the present study examined how the occupation specific stressor of worries about accidents and the individual resource attribute of PsyCap, are related to levels of sleepiness (i.e., difficulty in maintaining the wakeful state so that the person falls asleep if not actively kept aroused) among seafarers. Specifically, the aims of the study were to determine 1) whether worries about accidents are associated with increased sleepiness among seafarers, and 2) whether PsyCap is a protective resource that buffers this relationship.

\section{MATERIALS AND METHODS}

\section{DESIGN AND PROCEDURE}

The data was collected in 2012 from a Norwegian company in the offshore oil and gas industry. Questionnaires were sent to all workers employed in the company at the time of the survey. The total number of employees comprised 926 seafarers from 22 vessels operating in the North Sea and Southeastern Asia. Altogether 402 seafarers replied, giving a response rate of $43.3 \%$. Participation was voluntary and anonymous. The questionnaires were sent from the shipping company, answered by the seafarers when working at the vessels, and returned in sealed envelopes to the principal researcher. The questionnaires were written in Norwegian (for the Norwegian seafarers) and English (for all other seafarers). English is the work language in this shipping company. As all employees were invited to participate in the survey, the sampling is based on a probability mechanism. Respondents with more than $25 \%$ missing data on the study variables were excluded from the sample. Remaining missing data were replaced by the use of the Hot Deck imputation procedure [19]. Hot Deck imputation is a method for handling missing data in which each missing value is replaced with an observed response from a respondent with similar characteristic on pre-determined anchor variables. Age, years of experience at sea, and nationality (dichotomised into Western vs. Eastern country origin) was used as anchor variables in the Hot Deck imputation. The final sample comprised 397 respondents.

\section{SAMPLE}

Because there were very few women $(<1.0 \%)$ working on board the vessels, gender was not recorded in order to protect the anonymity of these women. The nationalities of the participants were Norwegian (34.9\%), other European countries (24.1\%), Filipino (36.7\%), and other Asian and Australasian countries (4.3\%). The age distribution of the seafarers was: under 25 years: $12.2 \%, 25-29$ years: $17.3 \%$, 30-39 years: $32.9 \%$, 40-54 years: $28.1 \%$, over 54 years: $9.5 \%$. In this sample $45.2 \%$ worked in deck detail, $26 \%$ in machine detail, $17.6 \%$ worked in gallery detail and $10.7 \%$ were captains $(0.5 \%$ did not report job title). The mean time the maritime workers had been working for the company was 3.73 years (range: 0-29 years). Their experience as seafarers ranged from 0-55 years, the mean was 14.78 years $(S D=10.95)$. Altogether $23.3 \%$ mainly worked night shifts, $44.1 \%$ mainly day shifts, and $32.5 \%$ worked another/not specified shift type.

\section{INSTRUMENTS}

Sleepiness. Sleepiness was measured with one of the subscales of the Swedish Occupational Fatigue Inventory (SOFI) [20-22]. The dimension describes feelings of sleepiness, and consists of four verbal expressions that describe how workers feel at the end of their work shift: falling asleep, drowsy, yawning, and sleepy. The participants were asked to answer how tired they usually feel after finishing their watch, and responses were recorded on a seven-point scale with anchors $1=$ Not at all and $7=$ To a very high degree. Cronbach's alpha for the scale was satisfactory (0.87). A confirmatory factor analysis (CFA) provided acceptable fit for the latent indicator (CMIN $=12.91 ; \mathrm{df}=1 ; \mathrm{CFI}=0.99 ; \mathrm{TLI}=0.91$ ). All factor loadings for the scale exceeded 0.66 .

Worries about accidents. Worries about prospective accidents were measured with a questionnaire based on the hazard categories used in official reports from the Norwegian Maritime Directorate [23]. The participants were asked to assess the probability for seven common accidents to happen to them during the next year: "Struck by objects", "Trapped, crushed or squeezed", "Cuts or contact with sharp or pointed elements", "Contact with hazardous substances", "Man over board", "Slips, trips, falls", and "Electrical hazard, fire, explosion". Answers were provided on a seven-point scale ranging from 1 = Very unlikely, through $4=$ Neither nor, to $7=$ Very likely. Cronbach's alpha for the scale was 0.90. A CFA provided acceptable fit for the latent indicator $(\mathrm{CMIN}=77.81 ; \mathrm{df}=7 ; \mathrm{CFI}=0.95 ; \mathrm{TLI}=0.89)$. 
Table 1. Testing the moderator effect of psychological capital on the relationship between worries about accidents (predictor) and sleepiness (outcome) using hierarchical multiple regression $(n=397)$

\begin{tabular}{|c|c|c|c|c|c|}
\hline Step and variable & B & SE B & $\beta$ & $\mathbf{R}^{2}$ & $\Delta \mathbf{R}^{2}$ \\
\hline Step 1 & & & & $0.167 * * *$ & \\
\hline Experience as seafarer [years] & -0.02 & 0.01 & $-0.12^{\star}$ & & \\
\hline Poor psychological safety climate & 0.29 & 0.11 & $0.14 * *$ & & \\
\hline Worries about accidents & 0.35 & 0.06 & $0.31 * * *$ & & \\
\hline Psychological capital (PsyCap) & -0.10 & 0.12 & -0.04 & & \\
\hline Step 2 & & & & $0.187 * * *$ & $0.02 * *$ \\
\hline Experience as seafarer (years) & -0.02 & 0.01 & $-0.13 * *$ & & \\
\hline Poor psychological safety climate & 0.27 & 0.11 & $0.13 *$ & & \\
\hline Worries about accidents & 0.34 & 0.06 & $0.30 * * *$ & & \\
\hline PsyCap & -0.14 & 0.12 & -0.06 & & \\
\hline Worries X PsyCap & 0.29 & 0.10 & $0.14 * *$ & & \\
\hline
\end{tabular}

PsyCap. PsyCap was measured with the 12-item Psychological Capital Questionnaire (PCQ-12) [11, 24]. The questionnaire consists of 12 statements about how the respondent is feeling right now about his/her job situation. The PCQ-12 items were extracted from the 24 item version of the PCQ, with 3 items for efficacy, 4 items for hope, 3 items for resiliency, and 2 items for optimism [12]. This shorter version of the PCQ was used to reduce the response burden of the participants. Answers were provided on a 6-point scale ranging from $1=$ Strongly disagree, to $6=$ Strongly agree . A confirmatory factor analyses of a four-dimension latent model had good fit to data (CMIN = 143.34; $\mathrm{df}=48$; $\mathrm{CFI}=0.96$; $\mathrm{TLI}=0.94)$. Factor loadings for all observed indicators exceeded 0.60. All four first order factors provided strong loadings when specified on a second higher order factor representing the overall PsyCap construct $(\mathrm{CMIN}=149.68$; $\mathrm{df}=50 ; \mathrm{CFI}=0.96 ; \mathrm{TLI}=0.94)$. Cronbach's alpha for this composite scale was 0.90 .

Psychological safety climate. Psychological safety climate was included as a covariate in analyses and was measured with 16 items from the Safety Climate Questionnaire [25]. The psychological safety climate items cover a range of interaction modes between supervisors and group members that assess the management's priority of safety versus competing goals such as production speed or schedules [25]. The respondents are presented with different statements and rate their level of agreement on a 5-point scale ranging from 1 = Completely disagree, to 5 = Completely agree. Examples of these statements are: "My direct supervisor discusses how to improve safety with us", and "My direct supervisor is strict about working safely when we are tired or stressed". The scale was recoded so that a high score indicates a poor psychological safety climate. The internal consistency for the scale (Cronbach's alpha $=0.95)$ was excellent. A CFA indicated that the latent variable had good psychometric properties $(\mathrm{CMIN}=482.07$; $\mathrm{df}=103 ; \mathrm{CFI}=$ $0.92 ; \mathrm{TLI}=0.91$ ). Factor loadings for all observed indicators were above 0.53 .

\section{STATISTICAL ANALYSIS}

Statistical analyses were conducted with IBM SPSS 22.0 and SPSS AMOS 23.0. The level of significance was set to $p<0.05$. For all measurement inventories, summary scales were calculated on the basis of a mean score of their respective items. Psychometric properties of the measurement models for the scales were determined by means of confirmatory factor analyses in SPSS AMOS. A comparative fit index (CFI) and Tucker-Lewis Index (TLI) with values in the area of 0.90 to 0.95 were employed as indicators of good model fit [26]. To explore the hypotheses about main and moderating effects, we conducted a hierarchical regression analysis, to test for linear associations between worries about accidents and sleepiness, as well as the interactive effects of worries and PsyCap, with regard to sleepiness (Table 1). The recommendations provided by Baron and Kenny [27] were followed, and, in accordance with Aiken and West [28], the predictor variables were centred prior to the two-way interaction analysis. The SPSS macro "Interaction and simple slopes test with two continuous variables" by Jason T. Newsom (http://web .pdx.edu/ ${ }^{\sim}$ newsomj/) was used to generate the regression estimates, plots, and simple slopes analyses.

\section{RESULTS}

Descriptives, reliability coefficients, and intercorrelations for all study variables are presented in Table 2. VIF-indexes 


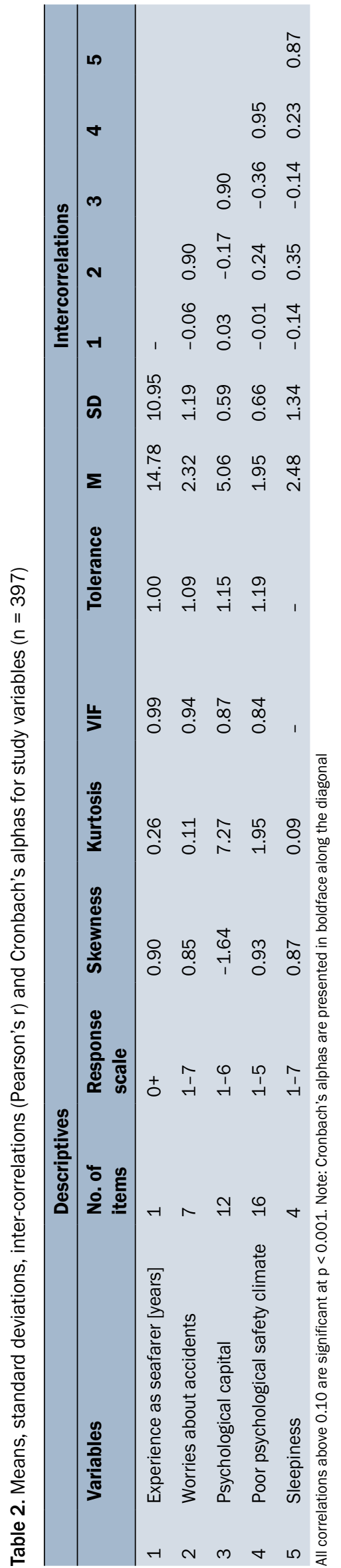

(range: 0.84-0.99) and tolerance values (range: 1.00-1.19) provided no indication of multicollinearity between the variables. Mean sleepiness score was 2.48 (SD = 1.34). This is perfectly in line with findings from previous studies that have used the same measurement inventory and response categories [29, 30]. The average score on poor psychological safety climate is also in line with previous research [31]. A mean value of 5.06 indicated that the level of PsyCap was high in the sample, while a standard deviation of 0.59 showed that the variation in PsyCap was low. A series of t-tests showed no differences between respondents from Eastern and Western countries with regard to worries about accidents $(t=1.81 ; d f=376 ; p>-0.05)$, PsyCap $(t=-1.65$; $\mathrm{df}=376 ; p>-0.05)$, poor psychological safety climate $(t=1.46 ; d f=376 ; p>-0.05)$, or sleepiness $(t=1.75$; $d f=376 ; p>-0.05)$.

The intercorrelations showed that worries about accidents ( $r=35 ; p<0.001)$ and inferior psychological safety climate $(r=0.23 ; p<0.001)$ was positively correlated, whereas PsyCap was negatively correlated $(r=-0.14$; $p<0.001$ ), with sleepiness. Worries about accidents was positively correlated with perceptions of the psychological safety climate as poor $(r=0.24 ; p<0.001)$ and negatively correlated with PsyCap $(r=-0.17 ; p<0.001)$. A negative correlation was established between PsyCap and perceptions of the psychological safety climate as poor $(r=-0.36$; $p<0.001)$.

\section{MAIN AND INTERACTION EFFECTS}

Findings from the multiple regression analyses of linear associations and interaction effects are presented in Table 1. All analyses were adjusted for years of experience as seafarer and poor psychological safety climate. For the linear association, the predictor and control variables explained $16.7 \%$ of the variance in sleepiness $\left(R^{2}=0.167 ; p<0.001\right)$. Worries about accidents $(\beta=0.31 ; p<0.001)$, experience as seafarer $(\beta=-0.12 ; p<0.05)$, and poor psychological safety climate $(\beta=0.14 ; p<0.01)$, but not PsyCap $(\beta=-0.10$; $p>0.05)$ yielded significant contributions to explaining the variance $(F=18.70 ; d f=4 ; p<0.001)$.

The finding of a negative relationship between worries about accidents and sleepiness supported the first study hypothesis. When adding the interaction term to the regression analysis, the amount of explained variance increased significantly by two percentage points $\left(R^{2}=0.187\right.$; $\left.p<0.01\right)$. As displayed in Table 1, the interaction made a significant contribution to the explained variance $\left(\beta=0.14 ; \Delta \mathrm{R}^{2}=\right.$ $=0.02 ; p<0.01)$, and the interaction model was significant ( $F=17.11 ; d f=5 ; p<0.001)$.

Thus, the findings show that PsyCap interacts with worries about accidents in explaining sleepiness. The associations between the other predictor variables and 


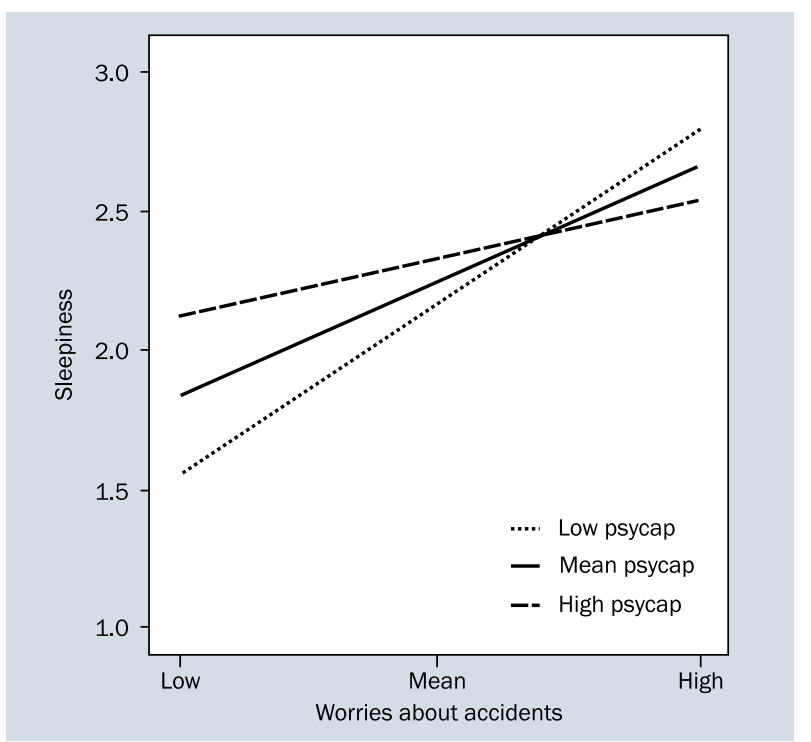

Figure 1. The interaction between worries about accidents and psychological capital (PsyCap) with regard to sleepiness. Low: -1 SD below mean, Mean: at mean, High: +1 SD above mean

sleepiness did not change after adding the interaction term. To examine the form of the interaction, a graphical display was created. Scores were plotted at the mean, low (1 SD below the mean) and high (1 SD above the mean) values on the predictor variables. As shown in Figure 1, the results indicate a stronger relationship between worries about accidents and sleepiness for the mean and high PsyCap groups than for the low PsyCap group. Follow-up analyses of simple slopes revealed that higher levels of worries about accidents were related to significantly elevated sleepiness among seafarers with a mean $(\beta=0.30$; $\mathrm{p}<0.01)$ and high PsyCap $(\beta=0.46 ; p<0.001)$, but not for seafarers with a low PsyCap $(\beta=0.15 ; p>0.05)$. Consequently, in direct contrast to our second hypothesis about a protective effect of PsyCap, the results indicate a reverse buffer association where PsyCap only seems to have a protective effect on the relationship between worries about accidents and sleepiness when levels of worries are low. When workers become more worried about accidents, there is a stronger increase in sleepiness among seafarers with high PsyCap compared to seafarers with low PsyCap.

The tests of interaction effects were replicated in a series of additional analyses where each of the four components of PsyCap was specified as a moderator. The finding showed that worries about accidents interacted significantly with self-efficacy $\left(\beta=0.27 ; \Delta R^{2}=0.03 ; p<0.01\right)$ and hope $\left(\beta=0.29 ; \Delta R^{2}=0.03 ; p<0.01\right)$, but not resiliency $(\beta=0.12$; $\left.\Delta R^{2}=0.01 ; p>0.05\right)$ or optimism $\left(\beta=0.11 ; \Delta R^{2}=0.01\right.$; $p>0.05$ ). The form of the significant interactions replicated the main findings of a reverse buffering effect.

\section{DISCUSSION}

The aims of this study were to examine the relationship between worries about prospective accidents and sleepiness among seafarers, and to examine PsyCap as a protective factor in this relationship. The present study adds additional empirical support to the assumption that worries about accidents constitutes a significant work-related stressor in that levels of worries were associated with increased sleepiness. However, the findings did not support the theoretical assumption of PsyCap as a protective resource. Contrary to our expectations, we found a reverse buffering effect where PsyCap only moderated the association between worries about accidents and sleepiness when levels of worries were low. When levels of worries were high, sleepiness was most prominent among workers with high PsyCap. All findings were controlled for experience as a seafarer and perceptions of psychological safety climate. With regard to those aspects of PsyCap that had the most important moderating effects, additional analyses showed that the interaction effect was only significant for self-efficacy and hope.

Although this latter finding may be counterintuitive to the common assumption that a robust personality should be protective with regard to the outcomes of work-related exposures, it may actually be that this awareness about risk may be highly beneficial in a maritime setting. That is, as PsyCap seems to enhance levels of sleepiness among high PsyCap workers in cases where they are very concerned about accidents, an interpretation is that workers with high PsyCap are especially sensitive to safety critical stimuli. When faced with substandard safety measures and safety critical operations high PsyCap workers may find themselves in a situation where they will be inclined to work to their limits in order to compensate for shortcomings in fellow crewmembers or equipment. Hence, the findings indicate that workers with high PsyCap do not necessarily see the world through rose-tinted glasses, but stay sensitive and vigilant to work-related risk factors. In short, these results show that workers with high levels of PsyCap will be attentive (as reflected through worrying) when the threat level is serious, but they will not be worrisome when exposed to the normal range of everyday strain and hassles associated with their work situation.

The above interpretation of our findings suggests that PsyCap is an individual asset that should be valued and even trained in the maritime industry. PsyCap has been highlighted as a malleable personality characteristic that is open to development and change [32], and organizations may therefore benefit from interventions that increase PsyCap among employees. As Schaubroeck and colleagues [18] have suggested, strategies can be developed to better shape the PsyCap dispositions among employees to facil- 
itate their coping with stress exposures. In maritime organizations timely and specific feedback on job performance may increase efficacy and self-confidence, while future goals and safety targets may increase hope and the ability to focus on safety at work. Furthermore, encouragement to keep up the performance despite setbacks may build resiliency and a realistic optimism about future accomplishments. After experiencing a risky situation on board a realistic optimist will analyse the situation and try to understand if the causes were personal or situational, permanent or temporary, and then make accurate causal attributions that fits this situation [12].

Despite the many favourable aspects of PsyCap in high risk situations, our findings also indicate that there may be thresholds with regard to how beneficial PsyCap will be in hazardous situations. That is, as extensive worrying over time seems to increase sleepiness, and there may be situations where heightened and prolonged sleepiness becomes unhealthy for the worker. It is therefore important to emphasize that the personal resources of PsyCap in themselves may not protect workers from sleep problems if there is substantial shortcomings in safety on board. In these cases, other organisational factors such as leadership practices in communicating risk and prioritizing safety are also important along with proper maintenance and training in emergency procedures and adherence to safety routines.

\section{METHODOLOGICAL IMPLICATIONS}

A notable strength of this study is that it is based on a relatively large and randomly drawn sample, applying internationally recognized instruments with satisfactory psychometric properties, which strengthen the validity of the findings. Nonetheless, some caution is needed when interpreting the results from the study. Firstly, the data is based on self-reports, with common-method variance as a possible problem [33]. Secondly, the data was cross-sectional, which implies that one cannot draw conclusions about causal relationships. That is, while this study focuses on the potential impact of worries on sleep, one cannot rule out the fact that there also is a reciprocal relationship between safety-related worries and sleep problems where poor sleep leads to more worries [34]. Longitudinal studies should be conducted to attain more knowledge about the causality of the relationships between worries about accidents, sleepiness, and PsyCap. Response bias could also be an issue because of factors like cross-cultural differences. The sample is from one company, but the seafarers are from many different national cultures, which may influence their individual interpretation of the questions.

It should be noted that PsyCap may be influenced by specific coping styles such as problem focused or appraisal coping, or other personality characteristics, such as neu- roticism, agreeableness, extraversion, conscientiousness, and openness. Hence, a limitation of the current study is that we did not examine and adjust for other personality characteristics and coping strategies. Furthermore, as the study was limited to worries about accidents we have not been able to adjust for the impact of other important work characteristics, such as job demands, leadership, and role expectations, that may influence sleepiness [6].

\section{CONCLUSIONS AND PRACTICAL IMPLICATIONS}

Extending previous research on PsyCap in safety critical organizations [17, 35, 36], our findings show that PsyCap serves as a buffer against worries about accidents in situations where levels of perceived risks are low, but not in cases where levels of perceived risks is high. On the one hand, this suggests that upcoming research could look into other factors that may act as stress buffers in high risk situations. One such factor could be safety specific leadership, as it is important to have a good operational leader in a high risk situation [37]. On the other hand, the fact that workers with PsyCap seem to react to worries about risks and accidents is a beneficial trait as this indicates that they are sensitive and vigilant with regard to risky situations at work. Hence, selecting, recruiting and training employees with high levels of PsyCap, and especially self-efficacy and hope, may be favourable with regard to preventing accidents in the maritime industry.

It should be noted that our study represents a single contribution to the knowledge about PsyCap in the maritime industry; hence, the findings should be replicated in upcoming research. Future studies could examine worries about accidents, PsyCap and sleepiness in other maritime settings as well as in other safety critical organizations such as healthcare, the police, or the emergency services.

\section{REFERENCES}

1. Håvold Jl. Safety-culture in a Norwegian shipping company. J Safety Res. 2005; 36(5): 441-458, doi: 10.1016/j.jsr.2005.08.005, indexed in Pubmed:16310804.

2. Nielsen D. Deaths at sea-a study of fatalities on board Hong Kong-registered merchant ships (1986-95). Safety Science. 1999; 32(2-3): 121-141, doi:10.1016/s0925-7535(99)00016-8.

3. Åkerstedt T. Psychosocial stress and impaired sleep. Scand J Work Environ Health. 2006; 32(6): 493-501, doi: 10.5271/sjweh.1054, indexed in Pubmed: 17173205.

4. Pereira D, Meier LL, Elfering A. Short-term effects of social exclusion at work and worries on sleep. Stress Health. 2013; 29(3): 240-252, doi:10.1002/smi.2461, indexed in Pubmed: 23027673.

5. Pillai V, Drake CL. Sleep and repetitive thought: The role of rumination and worry in sleep disturbance. In: Babson KA, Feldner MT, editors. Sleep and affect Assessment, theory, and clinical implications. Elsevier, New York 2015: 201-225.

6. Vleeshouwers J, Knardahl S, Christensen JO. Effects of Psychological and Social Work Factors on Self-Reported Sleep Disturbance 
and Difficulties Initiating Sleep. Sleep. 2016; 39(4): 833-846, doi: 10.5665/sleep.5638, indexed in Pubmed: 26446114.

7. Lichstein KL, Rosenthal TL. Insomniacs' perceptions of cognitive versus somatic determinants of sleep disturbance. J Abnorm Psychol. 1980; 89(1): 105-107, doi: 10.1037//0021-843x.89.1.105, indexed in Pubmed: 7365114.

8. Akerstedt T, Kecklund G, Axelsson J. Impaired sleep after bedtime stress and worries. Biol Psychol. 2007; 76(3): 170173, doi:10.1016/j.biopsycho.2007.07.010, indexed in Pubmed: 17884278.

9. Lazarus RS, Folkman S. Stress, appraisal, and coping. Springer, New York 1984.

10. Luthans F, Avolio B, Avey J, et al. Positive psychological capital: measurement and relationship with performance and satisfaction. Personnel Psychol. 2007; 60(3): 541-572, doi: 10.1111/j. 1744-6570.2007.00083.x.

11. Luthans F, Youssef CM, Avolio BJ. Psychological capital. Developing the human competitive edge. Oxford University Press, New York 2007.

12. Luthans F, Youssef-Morgan CM, Avolio BJ. Psychological capital and beyond. Oxford University Press, New York 2015.

13. Bandura A. Self-efficacy: The exercise of control. Freeman, New York 1997.

14. Seligman MEP. Learned optimism. How to change your mind and your life. 2nd ed. Pocket Books, New York 1998.

15. Luthans F, Avey JB, Patera JL. Experimental Analysis of a Web-Based Training Intervention to Develop Positive Psychological Capital. Academy Management Learning Education. 2008; 7(2): 209-221, doi: 10.5465/amle.2008.32712618.

16. Luthans F, Vogelsang GR, Lester PB. Developing the psychological capital of resiliency. Hum Recour Dev Rev. 2006; 5(1): 25-44, doi:10.1177/1534484305285335.

17. Bergheim K, Nielsen M, Mearns K, et al. The relationship between psychological capital, job satisfaction, and safety perceptions in the maritime industry. Safety Sci. 2015; 74: 27-36, doi: 10.1016/j. ssci.2014.11.024.

18. Schaubroeck JM, Riolli LT, Peng AC, et al. Resilience to traumatic exposure among soldiers deployed in combat. J Occup Health Psychol. 2011; 16(1): 18-37, doi: 10.1037/a0021006, indexed in Pubmed: 21280942.

19. Myers T. Goodbye, Listwise Deletion: Presenting Hot Deck Imputation as an Easy and Effective Tool for Handling Missing Data. Commun Methods Meas. 2011; 5(4): 297-310, doi: 10.1080/19312458.2011.624490.

20. Ahsberg E. Dimensions of fatigue in different working populations. Scand J Psychol. 2000; 41(3): 231-241, doi: 10.1111/14679450.00192, indexed in Pubmed: 11041305.

21. Åhsberg E, Garnberale F, Kjellberg A. Perceived quality of fatigue during different occupational tasks Development of a questionnaire. Int J Ind Ergon. 1997; 20(2): 121-135, doi: 10.1016/s0169-8141(96)00044-3.

22. Åhsberg $\mathrm{E}$, Kecklund $\mathrm{G}$, Åkerstedt $\mathrm{T}$, et al. Shiftwork and different dimensions of fatigue. Int J Ind Ergon. 2000; 26(4): 457-465, doi: 10.1016/s0169-8141(00)00007-x.

23. Norwegian Maritime Directorate. Marine causalities 20002010. http://www.sjofartsdir.no/en/safety/accident-statistics/ marine-casualties-2000-2010/(2012).
24. Luthans F, Avolio BJ, Avey JB. Psychological Capital (PsyCap) Questionnaire (PCQ): Mind Garden Inc. 2007.

25. Zohar D, Luria G. A multilevel model of safety climate: cross-level relationships between organization and group-level climates. J Appl Psychol. 2005; 90(4): 616-628, doi: 10.1037/0021-9010.90.4.616, indexed in Pubmed: 16060782.

26. Hu L, Bentler P. Cutoff criteria for fit indexes in covariance structure analysis: Conventional criteria versus new alternatives. Struct Equ Model. 1999; 6(1): 1-55, doi: 10.1080/10705519909540118.

27. Baron RM, Kenny DA. The moderator-mediator variable distinction in social psychological research: conceptual, strategic, and statistical considerations. J Pers Soc Psychol. 1986; 51(6): 1173-1182, doi: 10.1037//0022-3514.51.6.1173, indexed in Pubmed: 3806354.

28. Aiken LS, West SG. Multiple regression: Testing and interpreting interactions. Sage, Newbury Park, CA 1991.

29. Gutiérrez JG, Jiménez B, Hernández E, et al. Spanish version of the Swedish Occupational Fatigue Inventory (SOFI): Factorial replication, reliability and validity. Int J Ind Ergon. 2005; 35(8): 737-746, doi: 10.1016/j.ergon.2005.02.007.

30. Johansson S, Ytterberg C, Back B, et al. The Swedish occupational fatigue inventory in people with multiple sclerosis. J Rehabil Med. 2008; 40(9): 737-743, doi: 10.2340/16501977-0236, indexed in Pubmed: 18843426.

31. Nielsen MB, Tvedt SD, Matthiesen SB. Prevalence and occupational predictors of psychological distress in the offshore petroleum industry: a prospective study. Int Arch Occup Environ Health. 2013; 86(8): 875-885, doi: 10.1007/s00420-012-0825-x, indexed in Pubmed: 23099441.

32. Luthans F, Youssef CA, Avolio BJ. Psychological capital. Developing human competitive edge. Oxford University Press, Oxford 2007.

33. Podsakoff PM, Mackenzie SB, Lee JY, et al. Common method biases in behavioral research: a critical review of the literature and recommended remedies. J Appl Psychol. 2003; 88(5): 879-903, doi: 10.1037/0021-9010.88.5.879, indexed in Pubmed: 14516251.

34. Hystad SW, Nielsen MB, Eid J. The impact of sleep quality, fatigue and safety climate on the perceptions of accident risk among seafarers. Eur Rev Appl Psychol. 2017; 67(5): 259-267, doi: 10.1016/j. erap.2017.08.003.

35. Bergheim K, Eid J, Hystad S, et al. The Role of Psychological Capital in Perception of Safety Climate Among Air Traffic Controllers. J Leadersh Organ Stud. 2013; 20(2): 232-241, doi: 10.1177/1548051813475483.

36. Hystad SW, Bartone PT, Eid J. Positive organizational behavior and safety in the offshore oil industry: Exploring the determinants of positive safety climate. J Posit Psychol. 2014; 9(1): 42-53, doi: 10.1080/17439760.2013.831467, indexed in Pubmed: 24454524.

37. Eid J, Mearns K, Larsson G, et al. Leadership, psychological capital and safety research: Conceptual issues and future research questions. Safety Sci. 2012; 50(1): 55-61, doi: 10.1016/j. ssci.2011.07.001. 